\title{
MONOTONE FUNGTIONS MAPPING \\ THE SET OF RATIONAL NUMBERS ON ITSELF
}

(IN MEMORIAM FELIX A. BEHREND)

B. H. NEUMANN and R. RADO

(received 25 October 1962)

1.

The functions $f$ defined by

$$
f(x)=\frac{x}{c x+1-c}
$$

or by

$$
f(x)=\frac{x-1}{c x-1}
$$

for $c$ rational and less than +1 map the set of rational numbers between 0 and 1 one-to-one onto itself; and they are the only fractional linear functions with this property. Miss Tekla Taylor recently raised the question * whether these are the only differentiable functions with the stated property. In the present note we show, by two different constructions, that the answer is negative; in each case much freedom remains, which could be used to make the functions in question have various additional properties.

\section{2.}

Let $P$ denote the set of all rational numbers, $R$ the set of all real numbers, and $C$ the set of all complex numbers.

THEOREM 1. There is a function $f: R \rightarrow R$ with the following properties.

(i) $f$ is differentiable and monotone increasing in $R$, in fact $f^{\prime}(x) \geqq 1$ for all real $x$;

(ii) $f(P)=P$, that is to say, $f$ maps the set of rational numbers onto itself;

(iii) $f$ is not (entire) linear, that is to say, to all $a, b \in R$ there is an $x \in R$ such that $f(x) \neq a x+b$;

* Oral communication. A related but simpler problem, proposed by D. G. Northcott and communicated to us by $I$. D. Macdonald, is solved in a note by Peter M. Neumann in INVARIANT [the journal of the Oxford University Invariant Society] 1, 8-11 (1961). Subsequently one of us jointly with $\mathrm{H}$. A. Heilbronn obtained a more general result (not published). 
(iv) $f$ is "locally linear" at every rational point, in the sense that to each $\rho \in P$ there are numbers $\alpha, \beta, \delta \in P, \delta>0$, such that for all $x \in[\rho-\delta, \rho+\delta]$,

$$
f(x)=\alpha x+\beta .
$$

An immediate consequence of (iv) is that $f$ is not properly fractional linear (that is of the form $f(x)=(a x+b) /(c x+d)$ with $c \neq 0)$ in any real open interval.

ThEOREM 2. There is a function $f: C \rightarrow C$ with the following properties.

(i) $f$ is differentiable on $C$, that is to say, an entire function;

(ii) $f(R)=R$ and $f(P)=P$, that is to say, $f$ maps the sets of real numbers and of rational numbers onto themselves;

(iii) $f$ is monotone increasing on the real line, in fact, $f^{\prime}(x) \geqq 1$ for all $x \in R$;

(iv) $f$ is not a polynomial.

The montoneity of these functions $f$ on $R$ implies that they map $R$, and thus also $P$, one-to-one. Miss Taylor's question is answered by the function $\varphi$ defined by

$$
\varphi(x)=\frac{f(x)-f(0)}{f(1)-f(0)}
$$

where $f$ is the function either of Theorem 1 or of Theorem 2.

The proof of Theorem 1 is quite simple and short and occupies $\S 3$. The proof of Theorem 2 is more elaborate, as it requires the construction of an analytic function, not just a real once differentiable function; it is given in $\S 4$.

\section{Proof of Theorem 1}

Let

$$
P=\left\{\rho_{0}, \rho_{1}, \rho_{2}, \cdots\right\}
$$

be an enumeration of the rational numbers. It is possible to define, by simultaneous induction, integers $\lambda(n) \geqq 0$, closed intervals $I_{n} \subset R$ of positive length and with irrational endpoints, rational numbers $\alpha_{n}, \beta_{n}$, and differentiable functions $f_{n}: R \rightarrow R$, for $n=0,1,2, \cdots$, such that, let us say

$$
\lambda(0)=0, I_{0}=\left[\rho_{0}-\sqrt{ } 2, \rho_{0}+\sqrt{ } 2\right], \alpha_{0}=2, \beta_{0}=0, f_{0}(x)=2 x
$$

and such that, further, with the abbreviation

$$
J_{n}=I_{0} \cup I_{1} \cup \cdots \cup I_{n-1},
$$

we have for $n=1,2,3, \cdots$ 


$$
\begin{gathered}
I_{n} \cap J_{n}=\emptyset, \\
f_{n}(x)=f_{n-1}(x) \text { for all } x \in J_{n}, \\
f_{n}(x)=\alpha_{n} x+\beta_{n} \text { for all } x \in I_{n}, \\
\alpha_{n} \neq \alpha_{m} \text { for } m<n, \\
\left|f_{n}^{\prime}(x)-f_{n-1}^{\prime}(x)\right|<2^{-n} \text { for all } x \in R .
\end{gathered}
$$

Finally, we stipulate that

(a) if $n=2,4,6, \cdots$ then $\lambda(n)$ is the least integer $\lambda \geqq 0$ such that $\rho_{\lambda} \notin J_{n}$, and $I_{n}$ is so chosen that $\rho_{\lambda(n)} \in I_{n}$;

(b) if $n=1,3,5, \cdots$ then $\lambda(n)$ is the least integer $\lambda \geqq 0$ such that $\rho_{\lambda} \notin f_{n-1}\left(J_{n}\right)$, and then $I_{n}, \alpha_{n}, \beta_{n}$ are so chosen that $\rho_{\lambda(n)} \in f_{n}\left(I_{n}\right)$.

We first remark that from the definition of $f_{0}$ and from (5) we have

$$
1<f_{n}^{\prime}(x)<3 \text { for } n=0,1,2, \cdots \text { and all } x \in R \text {; }
$$

moreover, still by (5), $\lim _{n \rightarrow \infty} f_{n}^{\prime}$ exists uniformly in $R$. Also, by repeated application of (2)

$$
f_{n}\left(\rho_{0}\right)=f_{0}\left(\rho_{0}\right) \text { for } n=0,1,2, \cdots,
$$

so that $\lim _{n \rightarrow \infty} f_{n}\left(\rho_{0}\right)$ (trivially) exists. Hence

$$
\lim _{n \rightarrow \infty} f_{n}=f
$$

exists on $R$ and is differentiable, and

$$
f^{\prime}(x)=\lim _{n \rightarrow \infty} f_{n}^{\prime}(x) \geqq 1 \text { for all } x \in R .
$$

This proves (i).

Next, if $\rho \in P$, then there is an integer $m \geqq 0$ such that $\rho \in I_{m}$, by (a). Then for all $n \geqq m$,

$$
f_{n}(\rho)=f_{m}(\rho)=\alpha_{m} \rho+\beta_{m} \in P,
$$

by repeated application of (2), (3), and the choice of $\alpha_{m}, \beta_{m} \in P$; thus

$$
f(\rho)=\lim _{n \rightarrow \infty} f_{n}(\rho)=f_{m}(\rho) \in P,
$$

and it follows that $f(P) \subseteq P$.

Again, if $\sigma \in P$, then there is an integer $k \geqq 0$ such that $\sigma \in f_{k}\left(I_{k}\right)$, by (b). Thus there is $\rho \in I_{k}$ such that

$$
\sigma=\alpha_{k} \rho+\beta_{k},
$$

and as $\alpha_{k} \neq 0$ - an obvious consequence of $(3)$ and (6) - we have $\rho \in P$. Again we have as before $f_{n}(\rho)=f_{k}(\rho)$ for $n \geqq k$, and 


$$
f(\rho)=\lim _{n \rightarrow \infty} f_{n}(\rho)=f_{k}(\rho)=\sigma,
$$

and it follows that $P \subseteq f(P)$, completing the proof of (ii). Property (iii) is a consequence of (4), and (iv) follows from the fact that the endpoints of all $I_{n}$ have been chosen irrational. This completes the proof of Theorem 1 .

\section{Proof of Theorem 2}

We adopt the convention that $x$ ranges over the real numbers, $z$ over the complex numbers, and $m, n$ over the non-negative integers.

Let two sequences of (not necessarily distinct) rational numbers

$$
\pi_{0}=0, \pi_{1}, \pi_{2}, \cdots \text { and } a_{0}=2, a_{1}, a_{2}, \cdots
$$

be chosen so that for $m \geqq 1$

$$
-m \leqq \pi_{m} \leqq m \text { and } 0<a_{m} \leqq(2 m)^{-m^{2}-m-2} \text {. }
$$

We define polynomials $p_{0}, p_{1}, p_{2}, \cdots$ and $f_{0}, f_{1}, f_{2}, \cdots$ by

$$
\begin{aligned}
p_{m}(z) & =z^{m^{2}+1}\left(z-\pi_{1}\right)\left(z-\pi_{2}\right) \cdots\left(z-\pi_{m}\right), \\
f_{n} & =\sum_{m=0}^{n} a_{m} p_{m} .
\end{aligned}
$$

Then we have, for $m \geqq 1$ and $|z| \leqq m$,

$$
\begin{gathered}
\left|a_{m} p_{m}(z)\right| \leqq a_{m} m^{m^{2}+1}(2 m)^{m} \leqq 2^{-m^{2}} \leqq 2^{-m}, \\
\left|a_{m} p_{m}^{\prime}(z)\right| \leqq a_{m}\left(\left(m^{2}+1\right) m^{m^{2}}(2 m)^{m}+m^{m^{2}+1} m(2 m)^{m-1}\right) \\
\leqq a_{m} m^{m^{2}}(2 m)^{m+2} \leqq 2^{-m^{2}} \leqq 2^{-m} .
\end{gathered}
$$

Hence we may define a function $f: C \rightarrow C$ by

$$
f=\lim _{n \rightarrow \infty} f_{n}=\sum_{m=0}^{\infty} a_{m} p_{m}
$$

and as (9) ensures the uniform convergence in every circle, this function is entire. Also, by (10), we have

$$
f^{\prime}=\lim _{n \rightarrow \infty} f_{n}^{\prime}=\sum_{m=0}^{\infty} a_{m} p_{m}^{\prime} .
$$

We note that the only powers of the variable that actually occur in $p_{m}$ are among those with exponents $m^{2}+1, m^{2}+2, \cdots, m^{2}+m+1$, and the last of these has coefficient 1 ; hence the different $p_{m}$ contribute distinct powers of the variable to $f$, and in the power series expansion of $f$ about the origin, infinitely many powers occur with non-zero coefficients. It follows that $f$ is not a polynomial. 
Next we note that $p_{m}, p_{m+1}, p_{m+2}, \cdots$ all vanish at $z=\pi_{m}$, whence

$$
f\left(\pi_{m}\right)=f_{n}\left(\pi_{m}\right) \text { for } m \leqq n+1 \text {; }
$$

this is a rational number, and if we ensure that all rational numbers occur among the $\pi_{m}$, then we shall have $f(P) \subseteq P$.

However, before carrying this out, we prove that $f$ is monotone on the real line; to this end we show by induction that

$$
f_{n}^{\prime}(x) \geqq 1+2^{-n} \text { for all } x \in R .
$$

As $f_{0}=p_{0}$ is defined by $f_{0}(z)=2 z,(12)$ is true for $n=0$. Let now $m \geqq 0$ be fixed and assume (12) is valid for $n=m$. Then, if $|x| \leqq m+1$, we apply (10) and obtain

$$
f_{m+1}^{\prime}(x)=f_{m}^{\prime}(x)+a_{m+1} p_{m+1}^{\prime}(x) \geqq 1+2^{-m}-2^{-m-1}=1+2^{-m-1} .
$$

If $|x|>m+1$, then $p_{m+1}^{\prime}(x)>0$ since $p_{m+1}$ is a monic polynomial of odd degree whose roots are real and contained in the interval $[-m-1, m+1]$. Hence

$$
f_{m+1}^{\prime}(x)=f_{m}^{\prime}(x)+a_{m+1} p_{m+1}^{\prime}(x) \geqq 1+2^{-m}+0 \geqq 1+2^{-m-1} .
$$

This proves (12) for $n=m+1$, and thus (12) is true for all $n$. The monotoneity of $f$ follows at once:

$$
f^{\prime}(x)=\lim _{n \rightarrow \infty} f_{n}^{\prime}(x) \geqq 1 \text { for all } x \in R .
$$

It only remains to specialize the sequences (7) of rational numbers, subject to $(8)$, so that

$$
f(P)=P .
$$

Let again, as in the proof of Theorem 1,

$$
P=\left\{\rho_{0}, \rho_{1}, \rho_{2}, \cdots\right\}
$$

be an enumeration of the rational numbers, which we now choose so that

$$
\left|\rho_{m}\right| \leqq m \text { for all } m \geqq 0 .
$$

Recall that $\pi_{0}=0$ and $a_{0}=2$, so that $p_{0}$ and $f_{0}$ are already given. The coefficients $a_{m}$ for $m \geqq 1$ can be chosen arbitrarily; let us put

$$
a_{m}=(2 m)^{-m^{2}-m-2} \text { for } m \geqq 1 .
$$

We define $\pi_{n}$ inductively as follows. Suppose $\pi_{0}, \pi_{1}, \cdots, \pi_{n-1}$ have been determined, where $n$ is fixed, $n \geqq 1$; then $p_{m}$ and $f_{m}$ are defined for $m<n$.

Case 1. Let $n=3 k+1$. We put $\pi_{n}=\rho_{k}$. Then $\left|\pi_{n}\right| \leqq k<n$, as required 
by $(8)$. We thus ensure that all rational numbers occur among the $\pi_{n}$, and thus that

$$
f(P) \subseteq P .
$$

Case 2. Let $n=3 k+2$; we then define both $\pi_{n}$ and $\pi_{n+1}$. Determine $\xi \in R$ from the equation

$$
f_{n-1}(\xi)=\rho_{k} .
$$

There is precisely one such $\xi$, and as $\left|\rho_{k}\right| \leqq k<n$, and as $f_{n-1}(0)=0$ and $f_{n-1}^{\prime}(x)>1$ for all $x \in R$ (see (12)), also $|\xi|<n$.

Case 2a. Let $\xi \in P$. Then we put $\pi_{n}=\pi_{n+1}=\xi$. Then $\left|\pi_{n}\right| \leqq n$ and $\left|\pi_{n+1}\right| \leqq n+1$, as required by (8); and, by (11),

$$
f\left(\pi_{n}\right)=f_{n-1}\left(\pi_{n}\right)=\rho_{k} .
$$

Case $2 b$. Let $\xi$ be irrational. Define a function $F$ by

$$
F(x)=\frac{f_{n-1}(x)-\rho_{k}}{a_{n} x^{2 n-1} p_{n-1}(x)}+x .
$$

This function exists and is continuous in a neighbourhood of $\xi$, that is for $|x-\xi|<\delta$ with a suitable $\delta>0$; and

$$
|F(\xi)|=|\xi|<n .
$$

Hence there is a $\rho \in P$ such that $|\rho|<n$ and $|F(\rho)|<n$. Note that also $F(\rho) \in P$. We put

$$
\pi_{n}=F(\rho), \quad \pi_{n+1}=\rho .
$$

Then $\left|\pi_{n}\right| \leqq n$ and $\left|\pi_{n+1}\right| \leqq n+1$, as required by (8); and

$$
\begin{gathered}
\pi_{n}=F(\rho)=\frac{f_{n-1}(\rho)-\rho_{k}}{a_{n} \rho^{2 n-1} p_{n-1}(\rho)}+\rho \\
\rho_{k}=f_{n-1}(\rho)+a_{n} \rho^{2 n-1} p_{n-1}(\rho)\left(\rho-\pi_{n}\right)=f_{n-1}(\rho)+a_{n} p_{n}(\rho)=f_{n}(\rho)
\end{gathered}
$$

thus, using (11),

$$
f\left(\pi_{n+1}\right)=f_{n}\left(\pi_{n+1}\right)=f_{n}(\rho)=\rho_{k} .
$$

Now (16) and (17) combine to show that every rational number is of the form

and so

$$
\rho_{k}=f\left(\pi_{3 k+2}\right) \text { or } \quad \rho_{k}=f\left(\pi_{3 k+3}\right),
$$

$$
P \subseteq f(P) .
$$

In conjunction with (15) this shows (13) and completes the proof of Theorem 2.

The Australian National University The University, Reading 